\title{
Knowledge and Attitude of dentists towards antibiotic use in children: A cross- sectional survey
}

\author{
Anjali Malik 1,*, Karan Sharma ${ }^{2}$, Vinod Sachdev ${ }^{3}$, Shivani Mathur ${ }^{4}$ \\ ${ }^{1}$ PG Student, ${ }^{2}$ Lecturer, ${ }^{3}$ Professor \& HOD, ${ }^{4}$ Associate Professor, Dept. of Pedodontics and Preventive Dentistry, I. T. S. Centre \\ of Dental Studies and Research, Muradnagar, Ghaziabad, Uttar Pradesh, India
}

*Corresponding Author:

Email: Anjali.malik011@gmail.com

\begin{abstract}
Introduction: Antibiotics form an important constituent of therapeutic clinical care in dentistry. However recent reports highlight a growing injudicious use and rise of antibiotic resistance, with 7\%-11\% of all common antibiotics prescribed by dentist alone. The American Academy and pediatric dentistry has regarding antibiotic usage laid down guidelines catering specifically to the needs of children. Thus, the objective of this study was to compare the antibiotic prescription pattern among dental practitioners and the awareness of antibiotic resistance in Delhi - NCR region.

Objective: To compare the antibiotic prescription pattern and the awareness of antibiotic resistance across various strata of dentists.

Materials and Method: A total of 120 dentists participated in the study. Inclusion criteria was willingness to participate and mentally competent to answer in written format, for a self-designed questionnaire containing open and closed ended questions. Chi- Square Test was applied.

Results: When comparisons were made between Interns and MDS it was found that there was a significant increase in the prescription of antibiotics among the MDS rather than Interns for most of the oral conditions except for a few conditions such as trauma (Interns $=6.7 \% \mathrm{MDS}=11.7 \%$ ). When asked about the exact dosage of medication to be provided to children only $3.3 \%$ did not know about it. Rest $41.7 \%$ Interns and $46.7 \%$ MDS prescribed it according to weight and age both $26.7 \%$ Interns and $35 \%$ MDS prescribed it according to weight alone, 13.3\% Interns and 16.7\% MDS according to age, 10\% Interns and 6.7\% MDS according to height only. For the management of oral conditions, it was found that the majority of dentists prescribed amoxicillin (73.3\% Interns and $85 \%$ MDS) as their first choice followed by alone or in combination metronidazole (6.7\% Interns and $16.7 \%$ MDS), cephalosporin (5.0\% Interns and 6.7\% MDS), azithromycin, ciprofloxacin and erythromycin.

Conclusion: The results of the study highlight prevailing attitudes among dentists prescribing antibiotics to children. There exists ambiguity over correct antibiotic usage which should be dealt with appropriate understanding of disease, immune system \& comprehensive knowledge of antibiotic medications.
\end{abstract}

\section{Introduction}

Antibiotics are essential and abundantly prescribed in daily dental practice because of their effectiveness and lifesaving benefits. However, the unnecessary use of antibiotics has been observed and has persisted as a major issue. Antibiotic overuse, incorrect dosing, and extended duration are some of the leading causes of antibiotic resistance. It has been found that dentists prescribe between $7 \%$ and $11 \%$ of all common antibiotics. ${ }^{1,2}$

The American Academy of Pediatric Dentistry (AAPD) has published guidelines for antibiotic use, recognizing the increasing prevalence of antibiotic resistant microorganisms ${ }^{1}$. The guidelines aim to provide guidance for the proper and judicious use of antibiotic therapy in the treatment of oral conditions in children. Pediatric patients require special dosing that is adjusted according to their body weight so that most common formula is $\mathrm{mg} / \mathrm{kg}$ of body weight for the dosage of antibiotic. $^{3}$

Over prescribing of antibiotics in children may be associated with unfavourable side effects ranging from gastrointestinal disturbances, fatal diarrheal cases and even anaphylactic shock ${ }^{4}$. It has been reported that the over prescription of antibiotics in children is leading to increased fatal diarrheal cases and a drastic change in the sensitiveness of gut flora. ${ }^{1}$ This growing problem has contributed significantly to the morbidity and mortality due to infectious diseases, with rising death rates for communicable diseases. ${ }^{5}$ The World Health Organization (WHO) recognized this global problem and announced the theme for the year 2011 as "Antibiotic resistance: No action today, No cure tomorrow". ${ }^{6}$

Exploration of scientific literature has revealed a scarcity of literature with trends of over prescription of antibiotics in the pediatric population, which provided an impetus to conduct this survey, the objective of which was to compare the antibiotic prescription pattern among dental practitioners (based on AAPD guidelines) and the awareness of antibiotic resistance among pediatric dentists. ${ }^{7}$ The increasing resistance problems of recent years are probably related to overuse or misuse of broadspectrum agents. $^{8}$

\section{Materials and Method}

This study was a cross-sectional survey conducted amongst dentists of Delhi NCR region. A validated, selfdesigned questionnaire was prepared to collect information regarding the prescription pattern of antibiotics among dentists. The questionnaire contained 
both open-ended and close-ended questions. A total number of 120 dentists were included in the survey. A written informed consent was obtained from all the participants. The questionnaire comprised information pertaining to the following: Qualification and work experience, most common antibiotic prescribed, duration and dosage, clinical conditions for which antibiotics were prescribed, awareness about antibiotic prophylaxis, awareness about antibiotic resistance and prescription of sugar free antimicrobials in children. The data obtained were compiled, tabulated, and subjected to statistical analysis. (Fig. 1) For the comparison of prescription pattern between interns and MDS, the chi-square test was used.

\section{QUESTIONNAIRE}

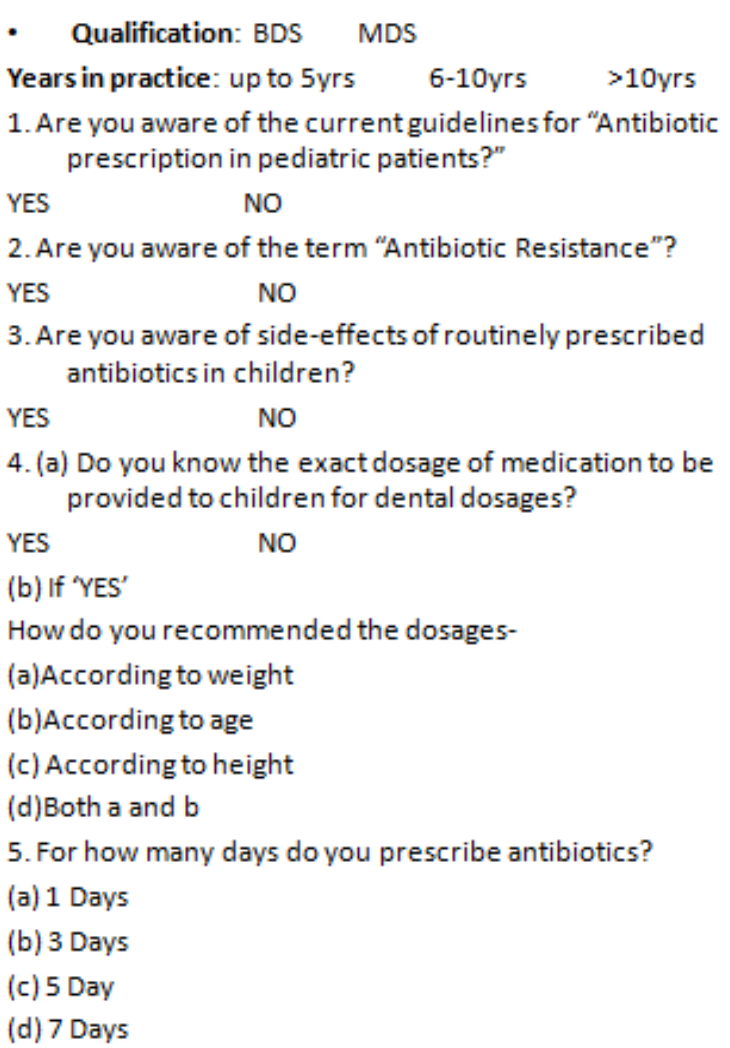

6. Which is the most commonly prescribed antibiotic by you?

(a)Amoxicillin

(b)Cephalosporin

(c)Metronidazole

(d) Erythromycin

(e)Ciprofloxacin

(f)Azithromycin

7. Do you frequently prefer, in routine practice, to compensate the questionable sterilization of instruments used procedure by giving more antibiotic cover?

YES NO

8. Are you familiar with the term "ANTIBIOMA"?

YES NO

9. Do you take history of any drug allergy in pediatric patients before prescribing medication?

YES NO

10. For which orodental conditions do you prescribe antibiotics in children -

(a)Oral-facial swelling

(b)Pain

(c) Trauma

(d) Dental caries

11. Do you feel, it is mandatory to prescribe antibiotic after every extractions?

YES NO

12. Do you prescribe sugar free antimicrobials in children?

YES

NO

Fig. 1: Questionnaire used in the study

\section{Results}

Out of total 120 dentists, 60 were INTERNS and 60 were MDS. The respondents in the prescribed time, filled and returned the Questionnaire (Figure1), the results of which were in the following manner. (Table1) For the question asked on prescription pattern for commonly encountered oral pathologies, a majority of respondents (70.0\% MDS and $68.3 \%$ Interns) prescribed antibiotic for oral facial swelling while the least for dental caries for which only $1.7 \%$ of MDS practitioners prescribed antibiotics. Response towards the question asked about commonly prescribed antibiotics in children, the pearson chi-square was significant being 1.207.(Table 2)On being asked on the basis of prescription involving correct dosages for children $41.7 \%$ Interns and $46.7 \%$ MDS dentists believed in prescribing antibiotics according to weight and age of the child. The pearson chi-square value for this question was 3.468 which was statistically significant. With regard to the duration of antibiotic prescribed, $65 \%$ Interns prescribed for 3 days, whereas $81.7 \%$ pediatric dentists (MDS) prescribed for 3 days, which was statistically significant. (Table 3) The pearson chi-square for this question was 7.675 which was statistically significant. For the management of oral conditions, it was found that the majority of dentists prescribed amoxicillin (73.3\% Interns and $85 \%$ MDS) as their first choice followed by metronidazole alone or in combination $(6.7 \%$ Interns and $16.7 \% \mathrm{MDS})$, cephalosporin (5.0\% Interns and 6.7\% MDS), followed by azithromycin $(3.3 \% \mathrm{MDS})$ and ciprofloxacin $(1.7 \%$ Interns) and erythromycin (1.7\% MDS) respectively (Table 4). Pearson chi-square for this question was 7.230 which was statistically significant. 
Table 1: Antibiotics prescribed for common Oro- facial conditions in children

\begin{tabular}{|l|c|c|c|c|}
\hline \multicolumn{1}{|c|}{ Category } & \multicolumn{2}{c|}{ Interns } & \multicolumn{2}{c|}{ MDS } \\
\hline \multicolumn{1}{|c|}{ Pathology } & No. & \% Age & No. & \%Age \\
\hline Orofacial swelling(a) & 42 & $70.0 \%$ & 41 & $68.3 \%$ \\
\hline Pain(b) & 12 & $20.0 \%$ & 11 & $18.3 \%$ \\
\hline Trauma(c) & 4 & $6.7 \%$ & 7 & $11.7 \%$ \\
\hline Dental caries(d) & 2 & $3.3 \%$ & 1 & $1.7 \%$ \\
\hline
\end{tabular}

Table 2: Correct dosage for children

\begin{tabular}{|l|c|c|c|c|}
\hline \multicolumn{1}{|c|}{ Category } & \multicolumn{2}{c|}{ Interns } & \multicolumn{2}{c|}{ MDS } \\
\hline \multicolumn{1}{|c|}{ Recommendation } & No. & \% Age & No. & \% Age \\
\hline According to weight(a) & 0 & $0 \%$ & 2 & $3.3 \%$ \\
\hline According to age(b) & 21 & $35.0 \%$ & 16 & $26.7 \%$ \\
\hline According to height(c) & 8 & $13.3 \%$ & 10 & $16.7 \%$ \\
\hline Both a and b(d) & 6 & $10.0 \%$ & 4 & $10.7 \%$ \\
\hline
\end{tabular}

Table 3: Duration of antibiotic Prescription

\begin{tabular}{|l|c|c|c|c|}
\hline \multicolumn{1}{|c|}{ Category } & \multicolumn{2}{|c|}{ Interns } & \multicolumn{2}{c|}{ MDS } \\
\hline No. of Days & No. & \% Age & No. & \%Age \\
\hline 1 Days(a) & 1 & $1.7 \%$ & 1 & $1.7 \%$ \\
\hline 3 Days(b) & 39 & $65.0 \%$ & 49 & $81.7 \%$ \\
\hline 5 Days(c) & 19 & $31.7 \%$ & 7 & $11.7 \%$ \\
\hline 7 Days(d) & 1 & $1.7 \%$ & 3 & $5.0 \%$ \\
\hline
\end{tabular}

Table 4: Most commonly prescribed antibiotic by dentist

\begin{tabular}{|l|c|c|c|c|}
\hline \multicolumn{1}{|c|}{ Category } & \multicolumn{2}{c|}{ Interns } & \multicolumn{2}{c|}{ MDS } \\
\hline Name of Drug & No. & \% Age & No. & \%Age \\
\hline Cephalosporilin(a) & 44 & $73.3 \%$ & 51 & $85.0 \%$ \\
\hline Metronidazole(c) & 3 & $5.0 \%$ & 4 & $6.7 \%$ \\
\hline Erythromycin(d) & 10 & $6.7 \%$ & 4 & $16.7 \%$ \\
\hline Ciprofloxacin(e) & 1 & $1.7 \%$ & 0 & $.0 \%$ \\
\hline Azithromycin(f) & 2 & $.0 \%$ & 1 & $1.7 \%$ \\
\hline
\end{tabular}

\section{Discussion}

Most oral diseases presented to dentists are primarily inflammatory conditions. A considerable percentage of dental pain originates from acute and chronic infections of pulpal origin, which necessitates operative intervention rather than antibiotics. ${ }^{4,5}$

However the awareness of guidelines for prescription of antibiotics was found to be low among the interns (35\%) in contrast to the MDS practitioners (94\%), when a study was done regarding the awareness of current guidelines for antibiotic prescription in pediatric patients. This calls for more emphasis and education amongst the Interns and upcoming Dentists for the prevailing guidelines of antibiotic prescription in children.

The most commonly used antibiotics in pediatric dental practice are amoxicillin, metronidazole, cephalosporins, azithromycin, ciprofloxacin and erythromycin. In the present study it was observed that $68.3 \%$ of the interns and $70 \%$ of the pediatric dentists
(MDS) relied on Amoxicillin as the first choice which is in confluence with studies conducted by Karibasappa $^{6}$ Montgomery, $^{7}$ Gonul $^{8}$ and Gregoire. ${ }^{9}$ Further, in the presence of anaerobic infection metronidazole was given in combination with amoxicillin by $6.7 \%$ interns and $16.7 \%$ of pediatric dentists (MDS). Similar finding were reported by Karibasappa $^{6}$ and Gonul. ${ }^{8}$ Cephalosporin was the next choice (5.1\% interns and $6.7 \%$ of MDS). Similar results were obtained in study conducted by Karibasappa ${ }^{6}$ Gonul. ${ }^{8}$ Who suggested that ofloxacin, ornidazole and cephaxim were preferred after metronidazole. There was a statistically significant difference between the prescription of combination of amoxicillin and metronidazole amongst the two groups which could be attributed to lesser exposure of combinations among the intern group.

In the present study, it was observed that $81.7 \%$ of the pediatric dentists (MDS) prescribed antibiotics for 3 days while $65 \%$ of the interns, while $5 \%$ of MDS and $1.7 \%$ of the interns prescribed antibiotics for 7 
days.Rubenstein ${ }^{10}$ advocated short courses to long particularly when treating children as compliance with conventional regimen is usually poor.

The knowledge regarding the awareness of the term antibiotic resistance in both the groups was found to be adequate i.e. $96.7 \%$ of both the groups were known for this .Another alarming finding was found in interns $(86.7 \%)$ who admitted to practice prescription of antibiotic to compensate a nonsterilized instruments in comparison to MDS practitioner (23.3\%).

The reason to this could be attributed to instances where questionable sterility of a dental instrument is hurriedly compensated by injudicious antibiotic cover, even in those conditions which usually don't require any. ${ }^{10}$ However the results were not statistically significant.

Another distressing finding was that neither the MDS (30\%) nor the interns $(80 \%)$ were aware of the term sugar free antimicrobials and its prescription which made us realize that it needs a lot of effort and awareness among dentists especially the interns for the role and use of sugar free antibiotics in pediatric patients.

\section{Conclusion}

In the present study, for many oral conditions, there was an over -prescription in the Interns group when compared to the MDS group. This may have probably been due to inadequate understanding of the disease, less skill, and less competency in performing operative intervention measures. Regarding the use of metronidazole, the awareness pertaining to the anaerobic infection control was less in Interns which is also a significant finding in this study, as the freshly passed out graduates should be clinically oriented more towards such issues. It has to be understood that dental diseases are predominantly because of local factors; the mere removal of the local causative factors reduces the need for prescribing antibiotics considerably.

In conclusion, the prescribing practices of dentists can be improved by increasing awareness of the recommended guidelines among dental practitioners.

Prescription of antibiotics by interns should be carefully supervised by senior faculty members in order to reduce chances of inadvertent antibiotic prescription.

\section{References}

1. Konde S, Jairam LS, Peethambar P, Noojady RS. Antibiotic overusage and resistance: A cross-sectional survey among pediatric dentists J Ind Soc of Ped and Prev Dent 2016;34(2):145-51.

2. Cherry WR. Cherry. American Academy on Pediatric Dentistry Council on Clinical Affairs. Guideline on appropriate use of antibiotic therapy for pediatric dental patients. Pediatr Dent 2008-2009;11:225-7

3. Huang Y, Mingyu J, Ren Z, Yang W.Knowledge, attitude and practice of antibiotics: a questionnaire study among 2500 Chinese students. BMC Med Educ 2013;13:163
4. Patait M, Urvashi N, Rajderkar M, Kedar S. Antibiotic prescription: An oral physician's point of view. J Pharm Bioallied Sci 2015;7(2):116-20.

5. Saeed Dar-Odeh N, Abu-Hammad O A, Al-Omiri MK. Antibiotic prescribing practices by dentists: a review. Ther Clin Risk Manag 2010;6:301-6.

6. Karibasappa GN, Sujatha A. Antibiotic resistance-a concern for dentists? IOSR-JDMS 2013;13:112-8

7. Montgomery EH, Kroeger DC. Use of antibiotics in dental practice. Dent Clin North Am 1984;28:433-53.

8. Gonul O, Aktop S, Satilmis T, Garip H, Goker K. In: Motamedi MH, editor. Odontogenic infections. A Textbook of Advanced Oral and Maxillofacial Surgery. Turkey:In Tech;2013.

9. Gregoire C. How are odontogenic infections best managed?J Can Dent Assoc 2010;76:114-6.

10. Rubeinstein E. British Dental Association; British Medical Association and royal pharmaceutical society of great Britain. Dental practitioner's formulary. London, UK: British Medical Association and the Royal pharmaceutical society of Great Britain;2006. 